\title{
LXVII. On the structure of the atom
}

\section{Sir J.J. Thomson O.M. F.R.S.}

To cite this article: Sir J.J. Thomson O.M. F.R.S. (1913) LXVII. On the structure of the atom , Philosophical Magazine Series 6, 26:154, 792-799, DOI: 10.1080/14786441308635024

To link to this article: http://dx.doi.org/10.1080/14786441308635024

$$
\text { 册 Published online: } 08 \text { Apr } 2009 .
$$

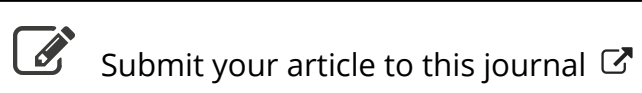

\footnotetext{
Џ Article views: 22
}

Q View related articles $\square$

4 Citing articles: 10 View citing articles $\square$ 
LXVII. On the Structure of the Atom.

$B y$ Sir J. J. Thomson, O.M., F.R.S.*

A I.ARGE and important class of atomic properties $A$ involves the relation $w=h n$ between the energy $w$ communicated to some of the corpuscles in the atom and the frequency $n$ of the light from which this energy is derived; $h$ is Planck's constant, which is the same for every kind of atom and on the C.G.S. system of units $=6.5 \times 10^{-27}$, its dimensions are those of a moment of momentum.

Examples of this type of properties are: (1) photo-electric effects, where the corpuscles ejected under the influence of. this light start with an amount of energy equal to $h n ;(2)$ the relation between the "hardness" of Röntgen rays and the kinetic energy of the cathode rays which produce them ; (3) the nature of the "charncteristic" Röntgen radiation emitted by the atom and probably also that of its visible spectrum. In fact, all the effects produced by radiation, whether this be ordinary light or Röntgen rays, seem to be manifestations of this principle.

It is usual to regard Planck's equation as an indication that radiant energy is molecular in structure. I have suggested, in papers in the 'Proceedings of the Cambridge' Philosophical Society' (vol. xvi. p. 643) and the 'Philosophical Magazine' (May, 1912), that the same result would follow without any such assumption as to the character of radiant energy, if the mechanism in the atom by which the radiant energy is transformed to kinetic energy is such as to require the transference to the mechanism of a definite amount of energy, sufficient, for example to rupture some system, before the transference can take place; that, in fact, Planck's relation depends on the properties of the atom, the agent by which the energy is transformed, rather than upon the existence of a structure in the energy itself.

In the following paper I have attempted to describe an atom of such a kind that the transformation of radiant into kinetic energy would take place in accordance with Planck's law.

Phenomena which involve this law seem especially suitable for consideration in connexion with theories of the structure of the atom, for their general character is the same for all atoms, and thus the mechanism to which they are due must exist in every kind of atom, and must, therefore, be of fundamental importance in any theory of atomic structure.

* Communicated by the Author. Read at the British Association, Sept. 11, 1913. 
In considering the forces which may exist in the atom, we must remember that we cannot assume that the forces due to the charges of electricity inside the atom are of exactly the same character as those given by the ordinary laws of Electrostatics; these laws may merely represent the averag 9 effect of a large number of such charges, and in the process of averaging some of the peculiarities possessed by the individuals may disappear.

Thus it is possible that the force exerted by a single atomic charge may not spread out uniformly in all directions, but be concentrated along certain lines or cones. The lines of electric force springing from such a charge may not fill all the space around the charged particle; each particle may be the origin of a force which, instead of filling the space round it, may be confined to a narrow tube of force, ontside which the particle produces no effect. In fine, we may attribute to the forces inside the atom a different distribution and character from that which might be expected if the forces due to the atomic charges obeyed in all respects the ordinary laws of Electrostatics. The only criterion we need apply to the forces inside the atom is that the properties which the atom would possess in virtue of these forces should correspond to the actual properties of the atom.

We shall now endeavour to show that properties of the kind we are considering would be possessed by the atom, if the character of the forces acting on a corpuscle in the atom was as follows :-

(1) A radial repulsive force, varying inversely as the cube of the distance from the centre, diffused throughout the whole of the atom, combined with

(2) A radial attractive force, varying inversely as the square of the distance from the centre, confined to a limited number of radial tubes in the atom.

With regard to these forces, one varying inversely as the cube, the other as the square of the distance, we may point out that they are the forces which occur most frequently in ordinary Mechanics. It is not necessary to elaborate this point with regard to the inverse square law. The force varying inversely as the cube of the distance is of the same type as ordinary "centrifugal force." It is the most usual type of force due to the kinetic energy of the system, when we regard forces as due to the inertia of systems connected with the primary one. In fact, much the same effects as are produced by the force varying inversely as the cube of the distance would be obtained, if there were something in the structure of the atom which compelled the moment of 
momentum of a corpuscle about an axis in the atom to be an absolute constant whose value did not depend at all upon the nature of the atom. We shall not, however, at this stage enter into any consideration as to the origin of this force; we shall simply postulate its existence.

If a corpuscle at $\mathrm{P}$ were inside one of the tubes of attractive force inside the atom, it could be removed to an intinite distance (1) by moving it radially outwards and keeping it inside the tube of attractive force the whole way. If the attractive force on unit charge at a distance $r$ from the centre is $\mathrm{A} / \mathrm{r}^{2}$, the work required to move the corpuscle in this way from $r$ to an infinite distance is Ae/ $r$; the corpuscle could, however, be moved to an infinite distance in another way (2) by moring it sideways out of the tube at $P$ and then moving it outside the tube to an infinite distance-the latter process will absorb no work, as the attractive force vanishes outside the tube. By the Conservation of Energy the work must be the same whether we adopt process (1) or (2), hence the work required to move the corpuscle sideways out of the tabe at $\mathrm{P}$ must be equal to $\mathrm{A} e / r$.

A corpuscle can be in stable equilibrium when in a region where it is acted on by both the repulsive and the attractive forces. Let the repulsive force on unit charge at a distance $r=\mathrm{C} / r^{3}$, the attractive $\mathrm{A} / r^{2}$, then there will be equilibrium at a distance $a$ if

$$
\frac{\mathrm{C}}{a^{3}}=\frac{\mathrm{A}}{a^{2}}
$$

To show that the equilibrium is stable, suppose the particle is displaced radially through a distance $x$, so that $r=a+x$; then, if $m$ is the mass of a corpuscle, $e$ its charge, the equation of motion is

$$
\begin{aligned}
m \frac{d^{2} x}{d t^{2}} & =\frac{\mathrm{C} e}{(a+w)^{3}}-\frac{\mathrm{A} e}{(a+x)^{2}}, \\
& =\frac{\mathrm{C} e}{a^{3}}\left(1-\frac{3 x}{a}\right)-\frac{\mathrm{A} e}{a^{2}}\left(1-\frac{2 x}{a}\right), \\
& =-\frac{\mathrm{C} e x}{a^{4}} .
\end{aligned}
$$

Thus the motion is stable, and if $\mathrm{T}$ is the time of vibration

$$
\frac{2 \pi}{\mathrm{T}}=\sqrt{\frac{\mathrm{C} e}{m a^{4}}} .
$$

The work done by the repulsive force on a corpuscle when it moves from $r=a$ to $r=$ infinity is $\frac{C e}{2 a^{2}}$; hence we see that 
this work, which we shall denote by $w$, is proportional to $1 / \mathrm{T}$, the frequency of the vibration; if $1 / \mathrm{T}=n$, we have

$$
w=\pi \sqrt{\text { Cem }} . n \text {. }
$$

We shall choose $\mathrm{C}$ so that

$$
\pi \sqrt{\mathrm{Cem}}=h,
$$

where $h$ is Planck's constant $6.5 \times 10^{-27}$; putting $e=4.7 \times 10^{-10}$, $e_{i} / m=5.3 \times 10^{17}$, we find $\mathrm{C}=10^{-17}$. Let us now consider some of the properties which an atom in which there are forces of this kind would possess. Take, first, the photoelectric effect. Suppose light of frequency $n$ falls upon the atom, it will find some corpuscle with which it is in resonance and communicate energy to it. The corpuscle will not be able to get out of the tube of attractive force in which it is situated unless it receives sufficient energy to get sideways out of the tube. This amount of energy is, as we have seen, $\mathrm{A} e / a=\mathrm{C} e / a^{2}=2 w$. When the energy reaches this value the corpuscle gets out of the tube, its kinetic energy being exhausted in the process. It now comes ander the uncontrolled action of the repulsive force and acquires kinetic energy, the kinetic energy when the corpuscle leaves the atom being equal to the work done by the repulsive forces on the corpuscle as it moves from $r=a$ to $r=i n f i n i t y$; this work is equal to $\mathrm{Ce} / 2 \alpha^{2}=w$, and this, as we have seen, is equal to $h n$, where $n$ is the frequency of the vibration and $h$ Planck's constant. Thus we see that the kinetic energy with which the corpuscle is expelled is proportional to the frequency of the light and is equal to the frequency multiplied by Planck's constant. This is the well-known law of Photo-Electricity.

Though the energy communicated to the corpuscle by the light would, in the first instance, appear as an increased radial velocity, we can easily conceive ways by which the radial velocity might, without loss of energy, be turned into a sideways velocity. For example, if when the corpuscle was passing through the position of equilibrium it came under the influence of some casual magnetic force at right angles to the direction in which it was moving, the ccrpuscle would be deflected and the radial velocity would be diminished, while the sideways velocity would increase without there being any change in the kinetic energy. Thus we see that it is quite possible for a corpuscle when acted upon by light to continually acquire kinetic energy without any commensurate increase in the amplitude of the radial displacement. 
An atom of this kind will only absorb radiant energy of frequency $n$ by multiples of $h n$. For unless the corpuscle is displaced from the tube in which it is situated there can be no real absorption of unergy; if a wave of light passed over it without displacing it from the tube it would leave the corpuscle vibrating with the same frequency as the light. 'The energy of this vibratory motion would be dissipated as electrical waves, $i$.e as light, so that the only result would be a scattering of the light without absorption. When, however, the energy given to the corpuscle is sufficient to liberate it from the tube there is a real absorption of energy as the particle ceases to vibrate, and its energy is now given out in the kinetic form. Thus the energy is absorbed by units, the unit being the amount of energy required to liberate a corpuscle from its tube-this, as we have seen, is equal to $2 h n$.

Next consider the conversion of potential into radiant energy by an atom of this kind. We regard the light as produced by a corpuscle falling from outside the atom into its position of equilibrium. It will arrive at this position with a certain amount of energy, which will be dissipated as light as the corpuscle vibrates about its position of equilibrium with the frequency $n$.

The kinetic energy possessed by the corpuscle when it falls to its position of equilibrium is

$$
\begin{array}{cc} 
& \mathrm{A} e \\
\text { since } & -\frac{1}{2} \frac{\mathrm{C} e}{2}=\frac{1}{a^{2}}=\frac{\mathrm{C} e}{2} a^{2}, \\
\frac{\mathrm{A}}{a}=\frac{\mathrm{C}}{a^{2}} .
\end{array}
$$

If we call this energy $w$ and $n$ the frequency of the vibration we have (see p. 795 )

$$
w=h n \text {. }
$$

Hence the potential energy of the corpuscle outside the atom is converted into thint of light of frequency $n$ by multiples of the unit $h n$. Hence we sue that an atom of the kind we are considering will possess the properties of the real atom with respect to the phenomena which involve transferences to and fro between radiant and kinetic or potential energy.

The case for the existence of a repulsive force varying inversely as the cube of the distance is strengthened, I think, by the consideration of the theory of the production of Röntgen rays by cathode rays. 
A cathode ray striking against an atom will penetrate into it until stopped by the repulsive force, then from the action of this force it will be repelled and ejected from the atom. As long as the corpuscle is inside the atom it will be accelerated and will therefore emit radiation, this radiation constitutes the Röntgen rays.

If the cathode particle approaches the atom radially, its equation of motion is

$$
m \frac{d^{2} x}{d t^{2}}=\frac{C e}{x^{2}},
$$

where $x$ is the distance from the centre at the time $t$. Integrating this equation we get

$$
m\left(\frac{d x}{d t}\right)^{2}=-\frac{\mathrm{C} e}{x^{2}}+m V^{2},
$$

where $\mathrm{V}$ is the velocity of the corpuscle before it strikes the atom.

Integrating this equation, and taking $t=0$ when the corpuscle is at its least distance from the centre of the atom, we have

$$
x^{2}=\mathrm{V}^{2} t^{2}+\frac{\mathrm{C}_{e}}{m \mathrm{~V}^{2}}=\mathrm{V}^{2}\left(t^{2}+\frac{\mathrm{C} e}{m \mathrm{~V}^{4}}\right) .
$$

We see from this that the time during which the cathode particle is in the neighbourhood of the place where it is nearest to the centre of the atom (i.e. the time when its acceleration is greatest, and its radiation therefore the most vigorous) is measured by

$$
\sqrt{\frac{\mathrm{C} e}{m V^{4}}}=\frac{1}{m V^{2}} \sqrt{\mathrm{Cem}}=\frac{h}{\pi m \nabla^{2}} .
$$

If we take $2 \pi$ times this time as corresponding to the time of vibration of the Röntgen rays, $n$ the "frequency" of the rays will be given by the equation

$$
n=\frac{m \mathrm{~V}^{2}}{2 h} \quad \text { or } \quad \frac{m \mathrm{~V}^{2}}{2}=h n .
$$

Thus Planck's relation holds between the "frequency" of the Röntgen rays and the kinetic energy of the cathode particles which produce them.

Phil. Mag. S. 6. Vol. 26. No. 154. Oct. 1913. $3 \mathrm{H}$ 
The total amount of energy in the Röntgen radiation is proportional to

$$
\int_{0}^{\infty}\left(\frac{d^{2} x}{d t^{2}}\right)^{2} d t
$$

or since

$$
\frac{d^{2} x}{d t^{2}}=\frac{G e}{x^{3}}
$$

and

$$
x^{2}=\mathrm{V}^{2} t^{2}+\frac{\mathrm{C} e}{m \mathrm{~V}^{2}}
$$

the energy is proportional to

$$
\begin{aligned}
& \frac{1}{m^{2}} \int_{0}^{\infty} \frac{\mathrm{C}^{2} e^{2} d t}{\left(\mathrm{~V}^{2} t^{2}+\frac{\mathrm{Ce}}{m \mathrm{~V}^{2}}\right)^{3}} \\
& =\frac{3 \pi}{16} \frac{m V^{4}}{\sqrt{\mathrm{C} e m}} .
\end{aligned}
$$

Thus the energy in the Röntgen radiation is proportional to the fourth power of the velocity of the cathode rays which excites it; this result, which $I$ gave in a paper in the "Philosophical Magazine' (August 1907), has been confirmed by Mr. Whiddington's experiments.

We can get some idea of the magnitude of the attractive forces varying inversely as the square of the distance by considering the energy required to excite radiation in the atom. We assume that the mechanism of radiation is somewhat as follows. A corpuscle is driven out from a position of equilibrium in the atom, leaving such a position vacant; then either this corpuscle or some other falls into the vacant place, vibrates about the position of equilibrium, and dissipates the hinetic energy it has acquired by falling into it as radiant energy. Thus, to excite the vibration, we have to drive a corpuscle out of its position of equilibrium; if this is at a distance $a$ from the centre of the atom, the work required is $\mathrm{A} e / a$, and since $a=\mathrm{O} / \mathrm{A}$ this is equal to $\mathrm{A}^{2} e / \mathrm{C}$.

Mr. Whiddington's experiments (Proc. Roy. Soc. A. vol. lxxxv. p. 323) show that, to excite the hardest characteristic Röntgen radiation, the $\mathrm{K}$ radiation, in an atom whose atomic weight is $\approx$, the cathode particle must have a velocity not less than $10^{8} \approx \mathrm{cm}$. $/ \mathrm{sec}$. The kinetic energy of one of these particles is thus not less than $\frac{1}{2} 10^{16} \varpi^{2} \mathrm{~m}$; equating this to $\mathrm{A}^{2} e / \mathrm{C}$, we get

$$
\frac{1}{2} 10^{16} \varpi^{2} m=\frac{A^{2} e}{C^{-}} .
$$


Since $e / m=5 \cdot 3 \times 10^{17}, C=10^{-17}$, we find

$$
\mathrm{A}=\varpi \times 3 \cdot 1 \times 10^{-10} \text {. }
$$

The charge on a corpuscle is $4.7 \times 10^{-10}$; the force at a distance $r$, calculated by the ordinary laws of Electrosiatics due to a charge $\varpi e$, is $\omega .4 .7 \times 10^{-10} / r^{2}$. We see, then, that the force along one of the tubes which contain the corpuscles which give the hardest characteristic Röntgen radiation, the $K$ radiation, when they vibrate, is about the same in magnitude as would, according to the ordinary laws of Electrostatics, be produced by a charge of positive electricity between $\frac{1}{2} \varpi e$ and $\varpi e$. If Whiddington's value for the velocity of the cathode particles is too great by about 20 per cent. the charge would be $\frac{1}{2} \omega e$, if it were too small by about 50 per cent. the charge would be $\varpi e$. The distance from the centre of the atom at which the corpuscles which produce these hard rays are situated, is C/A or

$$
\frac{1}{\sigma} 3 \cdot 2 \times 10^{-8} \mathrm{~cm} \text {. }
$$

Let us apply this result to the case of hydrogen; we shall suppose that in this case $\varpi e=\mathrm{A}$ or, since $\varpi=1, A=e$. The frequency of the characteristic radiation is (see p. 794) given by the equation

$$
4 \pi^{2} n^{2}=\frac{\mathrm{C} e}{m a^{4}}=\frac{e}{m} \frac{\mathrm{A}^{4}}{\mathrm{C}^{3}},
$$

since $a=\mathrm{C} / \mathrm{A}$. find

Putting $\mathrm{A}=e$, and substituting the values for $\mathrm{C}$ and $e$, we

$$
n=8 \cdot 2 \times 10^{14} \text {. }
$$

This frequency corresponds to light just in the ultra-violet. The most interesting thing about this result is, however, that, within the errors of experiment, the frequency we have found coincides with that of the head of the series of hydrogen lines given by Balmer's law, so that this spectrum represents the $\mathrm{K}$ radiation for hydrogen. As hydrogen possesses in the Schumann region a spectrum of still greater frequency, it seems probable that the $K$ radiation may not be the hardest type of characteristic Röntgen radiation.

If for helium we assume $A=\frac{7}{2} \varpi e$, the corresponding value of $n$ would be $3.288 \times 10^{15}$; this corresponds to radiation in the Schumann region. For heavier elements the frequency would be of an order corresponding to Röntgen rays. 